\title{
Laser-induced spin dynamics in ferromagnetic (In,Mn)As at magnetic fields up to 7 T
}

\author{
R. R. Subkhangulov, ${ }^{1}$ H. Munekata, ${ }^{2}$ Th. Rasing, ${ }^{1}$ and A. V. Kimel ${ }^{1}$ \\ ${ }^{1}$ Radboud University Nijmegen, Institute for Molecules and Materials (IMM), Heyendaalseweg 135, 6525 AJ Nijmegen, The Netherlands \\ ${ }^{2}$ Imaging Science and Engineering Laboratory, Tokyo Institute of Technology, Yokohama, Kanagawa, Japan
}

(Received 25 September 2013; published 10 February 2014)

\begin{abstract}
Laser-induced spin dynamics in (In,Mn)As is studied in magnetic fields up to $7 \mathrm{~T}$. It is shown that a laser pulse can effectively excite homogenous spin precession in this compound at the frequency of the ferromagnetic resonance. Laser excitation of this resonance appears to be very ineffective if the applied magnetic field is below $1.5 \mathrm{~T}$ at $10 \mathrm{~K}$. Our analysis shows that the damping of the laser-induced spin precession is a function of magnetic field and reaches very high values below $1.5 \mathrm{~T}$.
\end{abstract}

DOI: 10.1103/PhysRevB.89.060402

PACS number(s): 75.50.Pp, 75.78.Jp, 78.66.Fd, 78.47.D-

The discovery of ferromagnetism in III-V (In,Mn)As and $(\mathrm{Ga}, \mathrm{Mn}) \mathrm{As}$ diluted magnetic semiconductors triggered many experimental and theoretical studies of the transport, magnetic, and optical phenomena in these compounds [1-3]. Particularly, the hole-mediated ferromagnetic exchange [4-6] in these semiconductors led to intense discussions about intriguing opportunities to control their magnetic properties via a modulation of the holes concentration by light, electrical bias, or design in nano- and microstructures [7-13].

Soon after the discovery, very promising experimental results on optical control of magnetism in both (In,Mn)As and (Ga,Mn)As were achieved with low-power continuous wave excitation [7,14-16]. These observations raise the question about the feasibility to manipulate their intrinsic magnetic properties, such as magnetization and magnetic anisotropy, in an ultrafast, i.e., (sub)picosecond way by means of femtosecond laser pulses. A demonstration of laser excitation of ferromagnetic resonance (FMR) would be clear evidence of such ultrafast laser control of magnetism. To date, the possibility of triggering spin precession, namely optically excited FMR, was demonstrated for $(\mathrm{Ga}, \mathrm{Mn}) \mathrm{As}$ in a number of experimental works [17-21].

Recently new approaches were implemented in order to attain ultrafast optical control of magnetism in (Ga,Mn)As [22-24]. Despite several thorough studies of electron and spin dynamics triggered in (In,Mn)As by a femtosecond laser pulse [25-27], no optically excited FMR has been reported for this ferromagnetic semiconductor until now. This led to the question about the origin of such a distinct behavior of (Ga,Mn)As and (In,Mn)As. Here we show that the origin of the difference is a large effective damping of the magnetic precession in (In,Mn)As. We thus provide a recipe for studying the ultrafast laser-induced magnetization dynamics in (In,Mn)As. Regarding substantially different electronic structures of (Ga,Mn)As $\left(E_{G} \approx 1.5 \mathrm{eV}\right)$ and (In,Mn)As $\left(E_{G} \approx 0.4 \mathrm{eV}\right)$, one might compare those with magnetic insulator and magnetic metal, respectively. Hence our finding significantly broadens the playground for the investigation of the novel mechanisms of ultrafast control of magnetism in ferromagnetic semiconductors.

The tested $\operatorname{In}_{1-x} \mathrm{Mn}_{x}$ As semiconductor sample with a thickness of $15 \mathrm{~nm}$ and $x \approx 0.048$ was grown on a $500 \mathrm{~nm}$ buffer layer of $\mathrm{AlSb}_{0.9} \mathrm{As}_{0.1}$ (indirect band gap energy is $2 \mathrm{eV}$ ) deposited on a GaAs substrate (001) [10]. It is expected to have a direct, narrow band gap of $E_{g} \sim 0.4 \mathrm{eV}$ at low temperatures. The SQUID measurements have confirmed the existence of ferromagnetic order with the Curie temperature of about $T_{C} \approx 50 \mathrm{~K}$, the saturation magnetization $M=28 \mathrm{emu} / \mathrm{cm}^{3}$, as well as uniaxial magnetic anisotropy with the easy axis along [110] crystallographic direction in the plane of the sample. The SQUID measurements also revealed the presence of the paramagnetic contribution below the Curie temperature. The saturation magnetization measured at $0.1 \mathrm{~T}$ allowed us to estimate the amount of $\mathrm{Mn}^{2+}$ ions participating in the formation of ferromagnetic order $x_{\mathrm{eff}} \approx M /\left(g S_{\mathrm{Mn}} N_{0} \mu_{B}\right) \approx$ 0.034 , where $S_{\mathrm{Mn}}, g$ are spin and the Lande $g$ factor of $\mathrm{Mn}^{2+}$ ions, respectively; $\mu_{B}$ is the Bohr magneton; and $N_{0}$ is cations concentration in the III-V matrix. We infer that the strain, which is often not completely homogeneous over the entire epitaxial layer, enhances spatial fluctuation in the distribution of substitutional and interstitial Mn ions, and hence causes electrical and magnetic fluctuations in the sample.

Because $\mathrm{Mn}^{2+}$ ions in the indium sites act as acceptors, (In,Mn)As has $p$-type conductivity. Concentration and mobility of holes estimated from the low-field Hall measurements in this sample at room temperature are about $1.2 \times 10^{21} \mathrm{~cm}^{-3}$ and $\sim 2 \mathrm{~cm}^{2} / \mathrm{V} \mathrm{s}$, respectively. The estimations were done neglecting the contribution of the anomalous Hall effect $[28,29]$. Such a high value of the hole concentration suggests that the sample is in the metallic regime. In this regime the valenceband holes mediate a ferromagnetic RKKY (Ruderman-KittelKasuya-Yosida) exchange interaction between the spins of the $\mathrm{Mn}^{2+}$ ions [4,30]. Taking into account the reduced energy scale in the narrow-gap semiconductors, the mechanism incorporating impurity-band holes reported for $(\mathrm{Ga}, \mathrm{Mn}) \mathrm{As}$ [11,31-33] would not be of close relevance in the case of our sample.

The magneto-optical measurements were performed using a superconducting split coil magneto-optical cryostat. The sample was mounted inside the cryostat such that the angle between the normal to the sample surface and the external magnetic field was about $45 \mathrm{deg}$ [Fig. 1(a)]. For time-resolved magneto-optical studies we employed $60 \mathrm{fs}$ laser pulses with a repetition rate of $1 \mathrm{kHz}$ and a pump-probe technique in which the intensity of the pump was about 50 times stronger than that of the probe. The wavelength of the probe and pump pulses was set to $800 \mathrm{~nm}$ (photon energy $1.55 \mathrm{eV}$ ), which is 

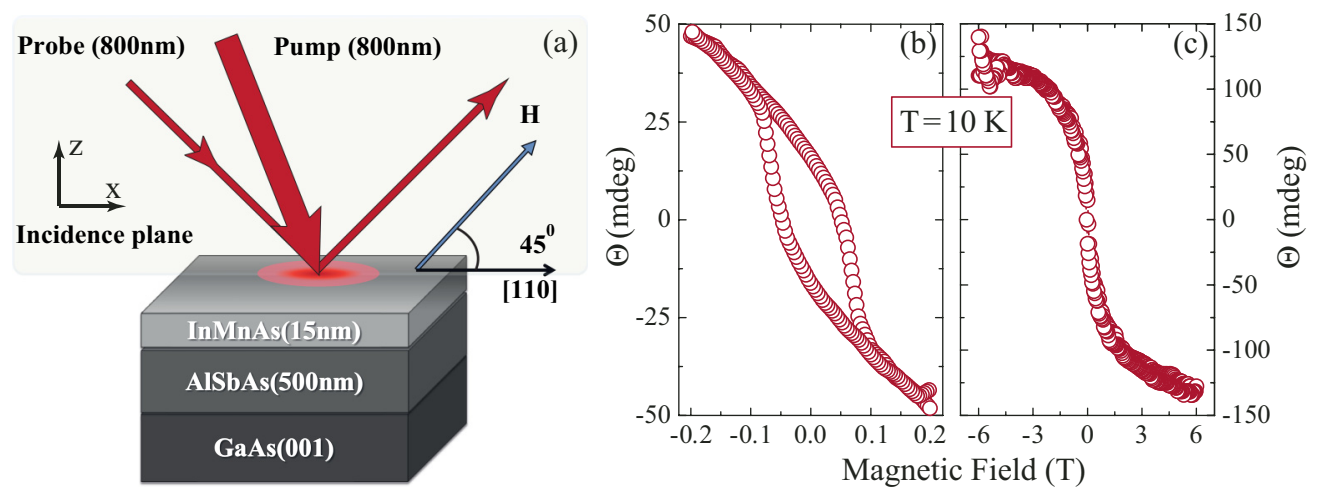

FIG. 1. (Color online) (a) Experimental geometry and description of the constituting sample layers. At some value of magnetic field the magnetization vector will be between the vector [110] and that of magnetic field. (b) Hysteresis loops in magneto-optical Kerr rotation measurements for a small (up to $0.2 \mathrm{~T}$ ) (b) and large (up to $6 \mathrm{~T}$ ) (c) ranges of magnetic fields.

higher than that of the band gap. The spot sizes were kept roughly $100-200 \mu \mathrm{m}^{2}$ for the probe and $400-1000 \mu \mathrm{m}^{2}$ for the pump. Pump fluences used in our experiment were in the range $20-1500 \mu \mathrm{J} / \mathrm{cm}^{2}$. The angle of incidence for the probe and pump beams were set to approximately 55 and $45 \mathrm{deg}$, respectively. Both external magnetic field and [110] axes lie in the plane of incidence of the pump and probe beams. These beams were $S$ polarized. To characterize the magneto-optical response from the material, we measured the polarization rotation $\Theta_{\text {Kerr }}$ of the probe upon reflection from the sample as a function of external magnetic field $\mathbf{H}$. The results obtained in fields up to 0.2 and $6 \mathrm{~T}$ [see Figs. 1(b) and 1(c)] show sensitivity of the magneto-optical technique to the net magnetization of the material. The chosen experimental geometry is sensitive to both the longitudinal and polar magneto-optical Kerr effect allowing us to probe in-plane and out-of-plane magnetization components.

To investigate laser-induced magnetization dynamics we measured the magneto-optical Kerr rotation for the probe as a function of delay between pump and probe pulses. To separate the magneto-optical Kerr rotation from other phenomena, which also may lead to a polarization rotation, we calculated the difference of the polarization rotation measured at two magnetic fields $H$ of opposite signs $\Delta \theta_{\text {Kerr }}=\Delta \theta(+H)-$ $\Delta \theta(-H)$. Figure 2 shows the dynamics of the magneto-optical Kerr rotation measured at different temperatures for a pump fluence of $0.25 \mathrm{~mJ} / \mathrm{cm}^{2}$ in a magnetic field $4 \mathrm{~T}$. It is seen that the laser excitation leads to an ultrafast decrease of the magneto-optical signal followed by its slow recovery. Such a dynamics is explained in terms of subpicosecond laser-induced demagnetization within $100 \mathrm{fs}$ and a recovery of the net magnetic moment at the time scale much larger than $100 \mathrm{ps}$ [27]. It should be noted that the demagnetization time is not dependent on the pump fluence while the degree of the demagnetization does increase with increasing fluence. The data also disclose that the pulsed excitation triggers oscillations of the magneto-optical signal. These oscillations, as it will be discussed later, are strongly damped. To disclose the origin of these oscillations, we fitted the measured signals with a function similar to the one obtained in [34] and retrieve the amplitude $A$, decay time $t$, and frequency $f$ of the oscillations.
The extracted frequency and amplitude of the oscillations are plotted in Figs. 2(b) and 2(c). Note that the frequency of the oscillations measured at field $4 \mathrm{~T}$ is close to that expected for the paramagnetic resonance of $\mathrm{Mn}^{2+}$ spins in II-VI semiconductors [35]. It is seen that the amplitude decreases with increasing bath temperature and vanishes above the Curie temperature. Such dependence reveals that the amplitude of the oscillations follows the behavior expected for the order parameter, i.e., the net magnetization of the medium. The presence of the magneto-optical signal above the Curie temperature [see Fig. 2(c)] is explained in terms of the smearing of the second order phase transition in the external magnetic field. Even if the sample contains paramagnetic regions which do not become ferromagnetic below $T_{C}$, such a temperature dependence clearly indicates that the observed oscillations must be associated with the spin precession in the ferromagnetic regions rather than paramagnetic ones.

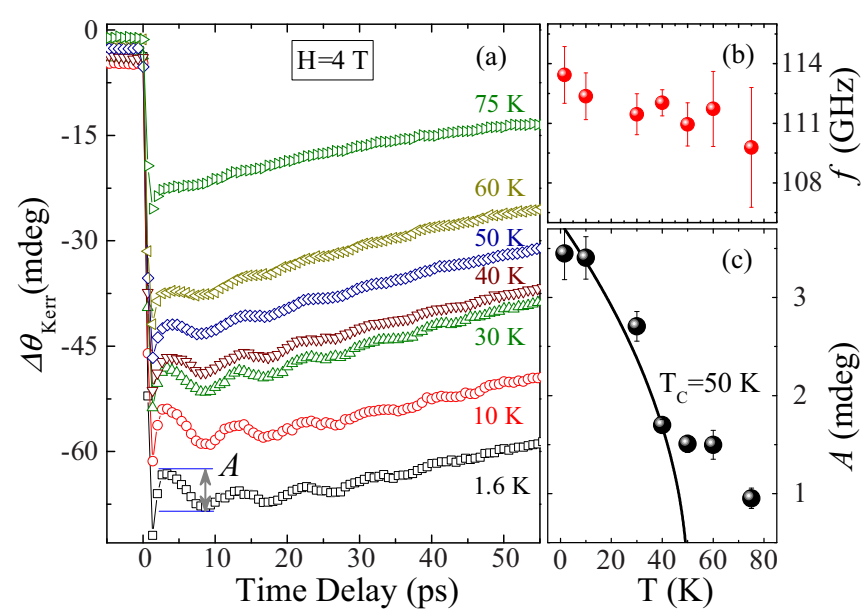

FIG. 2. (Color online) (a) Laser-induced dynamics of the magneto-optical Kerr rotation measured at different temperatures for a pump fluence of $0.25 \mathrm{~mJ} / \mathrm{cm}^{2}$ at $H=4 \mathrm{~T}$. (b) The frequency of the oscillations of the magneto-optical signal as function of temperature. (c) The amplitude of these oscillations as a function of temperature; dots are the experimental data line fit by the formula $A \sim\left(1-T / T_{C}\right)^{1 / 2}$, where $T_{C}$ is the Curie temperature. 


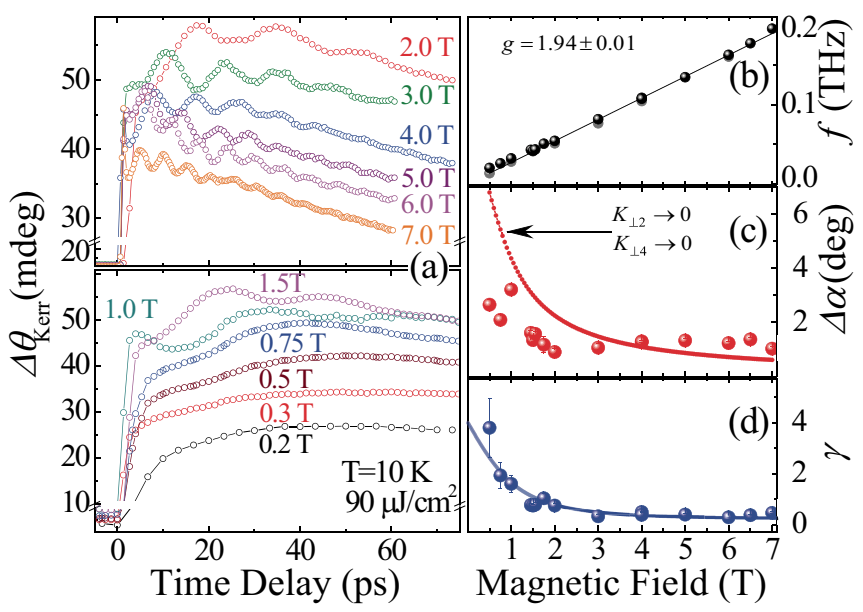

FIG. 3. (Color online) (a) Ultrafast dynamics of the magnetooptical Kerr rotation measured at different magnetic fields at $10 \mathrm{~K}$ and a pump fluence of $90 \mu \mathrm{J} / \mathrm{cm}^{2}$. (b) The frequency of the oscillations as a function of the magnetic field (dots are experimental data, solid line is linear fit). (c) Magnetization tilt angle as a function of the magnetic field (dots) in comparison with simulated deviation of the equilibrium orientation of the magnetization upon full quenching of the magnetic anisotropy (lines). (d) The damping of the oscillations as a function of the magnetic field.

Moreover, unlike the paramagnetic resonance, the frequency of the ferromagnetic resonance softens upon approaching the Curie temperature. Indeed an increase of temperature from 1.6 to $75 \mathrm{~K}$ results in a slight decrease of the frequency of the oscillations from 113 to $109 \mathrm{GHz}$. All these features imply that these oscillations are due to the optically induced ferromagnetic resonance of $\mathrm{Mn}^{2+}$-ordered spins in (In,Mn)As.

In order to substantiate this interpretation, measurements in magnetic fields up to $7 \mathrm{~T}$ at $10 \mathrm{~K}$ were performed with subsequent fit of the obtained data [see Fig. 3(a)]. Extracted frequencies $(f)$ and magnetization tilt angle $(\Delta \alpha)$ of the oscillations as well as their effective damping defined as $\gamma=1 /(f \cdot \tau)$ are depicted in Figs. 3(b)-3(d).

The frequencies obtained from the fit are plotted in Fig. 3(b). It is clearly seen that the frequency of the oscillations depends linearly on the external magnetic field. In particular, it can be described by the formula $\omega=g_{\text {eff }} \mu_{B} H / \hbar$, which is applicable for the case $H>>H_{A}$. Since the magnetic anisotropy field $H_{A}$ in the compound is expected to be 0.2-0.3 $\mathrm{T}$ [36], the linear dependence is not surprising. The fit of the frequency dependence on magnetic field yields a $g$ factor $g=1.94 \pm$ 0.01 . The estimated value of the $g$ factor is in good agreement with those obtained from conventional FMR measurements of (In,Mn)As and (Ga,Mn)As in the frequency domain [36-38]. The effective $g$ factor of the material can be treated as that for a ferrimagnet. For the FMR mode of a (In,Mn)As one can write

$$
g_{\text {eff }}=\frac{M_{\mathrm{Mn}}-M_{\mathrm{Mn}} x}{\frac{M_{\mathrm{Mn}}}{g_{\mathrm{Mn}}}-\frac{M_{\mathrm{Mn}} x}{g_{h}}}=\frac{1-x}{\frac{1}{g_{\mathrm{Mn}}}-\frac{x}{g_{h}}},
$$

where $g_{\mathrm{Mn}}, g_{h}$ are the $g$ factors of the $\mathrm{Mn}^{2+}$ and hole; $M_{\mathrm{Mn}}$ is the net magnetization of the $\mathrm{Mn}^{2+}$ ions; and $x$ is the fraction of the hole magnetization in comparison with the $\mathrm{Mn}^{2+}$. Taking the hole magnetization as $M_{\mathrm{Mn}} \cdot 0.05$ [38] and the $g$ factor of holes as that in (Ga,Mn)As $g_{h}=4-7$ [39,40], one gets that $g_{\text {eff }}=1.93-1.95$, showing a good agreement with the measured $g$ factor. Thus the decreased effective $g$ factor is likely due to the antiferromagnetic coupling between the spins of the free holes and the $\mathrm{Mn}^{2+}$ ions [38].

It is worth underlining that the amplitude of the FMR oscillations is rather large. One can estimate the magnetization tilt angle $\Delta \alpha$ to be as large as 3-4 deg, i.e., comparable with those obtained for (Ga,Mn)As [24] [see Fig. 3(c)]. The estimation is done using $\Delta \alpha=\arctan \left(A / \Theta_{\text {Kerr }}\right)$, where $A$ is the FMR amplitude extracted from fit [see Fig. 3(a)] and $\Theta_{\text {Kerr }}$ is a measure of the magnetization saturation obtained with the help of the static magneto-optical Kerr effect [see Fig. 1(b)].

Although the amplitude is large, the observation of the oscillations is substantially hampered by the high damping $\gamma$ [see Fig. 3(d)]. For the fields below 1.5 $\mathrm{T}$ the damping is so large that an effective laser excitation of the ferromagnetic resonance hardly occurs. The damping itself was found to be 60-240 times larger than that in (Ga,Mn)As $[18,19,21]$. We note that most of the measurements of the ultrafast laser-induced dynamics reported for (In,Mn)As so far have been performed in the range of magnetic fields where the effective damping is large and, consequently, observation of the ferromagnetic resonance was barely possible $[27,41]$.

Before explaining such a field-dependent damping of the laser-induced spin precession, first we identify the mechanisms of the laser excitation of the ferromagnetic resonance. To this end we performed measurements of the laser-induced dynamics for different polarization states of the pump pulses. No polarization dependence of the laser-induced spin dynamics was observed in the experiments, showing that a direct impact of light on the magnetization similar to that described by the inverse magneto-optical Faraday [42], Cotton-Mouton [43], and Kerr [44] effects can be excluded.

One of the possible mechanisms for ultrafast laser excitation of the ferromagnetic resonance is an ultrafast change of the equilibrium orientation of the magnetization. The latter is defined by the minimum of the free energy $W$ [45], which for the studied case can be written as

$$
\begin{aligned}
W= & \frac{-\sqrt{2} M H}{2}\left[\cos (\theta)+\sin (\theta) \cos \left(\phi-\frac{\pi}{4}\right)\right] \\
& -2 \pi M^{2} \sin ^{2}(\theta)-H_{2 \perp} M \cos ^{2}(\theta)-\frac{H_{4 \perp} M}{2} \cos ^{4}(\theta),
\end{aligned}
$$

where $M$ is the magnetization, $H$ is the external magnetic field, and $H_{2}, H_{4 \perp}$ are the out-of-plane uniaxial and cubic anisotropy fields, respectively. $\theta, \phi$ are angles which the magnetization forms with $z$ and $x$ axes, respectively. It is known that an ultrafast laser excitation of (In,Mn)As results in an ultrafast demagnetization of the material [27,41,46,47]. Such a demagnetization affects the balance between the terms in (2) leading to an ultrafast change of the equilibrium orientation of the magnetization and consequently to oscillations of the spins around the new equilibrium axis. Our experiments 
have indeed demonstrated such ultrafast demagnetization of (In,Mn)As up to $90 \%$. However, our simulations allow us to exclude that the spin oscillations are excited due to such an ultrafast demagnetization. Assuming that the effective fields of magnetic anisotropy are equal to those from Ref. [37], i.e., $0.2 \mathrm{~T}$ for the uniaxial anisotropy and $0.1 \mathrm{~T}$ for the cubic anisotropy, and taking the external magnetic field to be as low as $0.5 \mathrm{~T}$, we calculated that the equilibrium orientation of the magnetization changes only over 0.5 deg upon $90 \%$ demagnetization. This deviation becomes even less at higher magnetic fields.

Another possible scenario of the laser exited ferromagnetic resonance might be an ultrafast change of the magnetic anisotropy. Within the picture of hole-mediated ferromagnetism, magnetic anisotropy is modeled in terms of the spinorbit coupling in the valence band: the strength and direction of the anisotropy vary with both the effective temperature and numbers of holes in the valence bands $[6,48,49]$. Excitation of holes by photons with an energy higher than that of the band gap will result in an increase of both temperature and concentration, which in turn will change the magnetic anisotropy. Assuming that all the energy of the pump pulse is absorbed in (In,Mn)As one can estimate that the concentration of the photogenerated carriers is $\Delta n \sim 10^{19} \mathrm{~cm}^{-3}$. This might lead to considerable changes of the magnetic anisotropy. Suppose that the laser excitation just quenches the magnetic anisotropy. Such a quenching will change direction of the effective field and tilt the magnetization vector towards the magnetic field according to Eq. (2) and thus trigger magnetization precession around the new equilibrium. We calculated how the equilibrium orientation of the magnetization in (In,Mn)As changes upon the sudden and complete quenching of both the cubic and uniaxial magnetic anisotropies [Eq. (2)]. The result is shown in Fig. 3(c) by the bold line. Although the used model is rather simple it gives a good qualitative, and at high fields even quantitative, agreement with the experiment.

Finally, we discuss the mechanism of disappearance of an oscillatory magneto-optical signal in the region of low magnetic fields $(H<1.5 \mathrm{~T})$. The mechanism is based on the fact that ferromagnetic and paramagnetic regions coexist in the sample below the Curie temperature, as suggested experimentally by the present magneto-optical studies [see Figs. 1(b), 1(c), and Fig. 3(a)] and by the SQUID magnetometry measurements. That fact was observed previously [14,28,29,50-52]. It is known that the presence of impurities and inhomogeneities can open up additional channels for magnon-magnon scattering, thus leading to an increased effective damping of FMR [53]. At low fields, optically excited magnons with $\mathbf{k}=0$ created in the ferromagnetic region are scattered at the boundaries between these two regions, which results in the damping large enough to suppress the precession motion. When a strong magnetic field is applied, spins in the paramagnetic regions are aligned, giving rise to the disappearance of the boundaries between the regions, and thus reduction in damping. Indeed, in our experiments it is seen that the effective damping decreases up to a magnetic field of 1.5-2 $\mathrm{T}$ and hardly changes upon further increase. The values of the magnetic field at which the damping is saturated are in a good agreement with the magnetic field which aligns the paramagnetic regions in the material [see Fig. 1(c)].

According to [53], an increase of the effective damping due to magnetically disordered regions can be treated using the following equation:

$$
\frac{1}{\alpha} \sim \frac{V_{\mathrm{par}}}{V},
$$

where $\alpha$ is the effective damping of the magnetic oscillations and $V_{\mathrm{par}}, V$ are the volumes of the paramagnetic regions and sample volume, respectively. With $V_{\text {par }}$ scaling as a Brillouin function, this leads to a qualitative agreement with our observations.

The three processes of uniform mode scattering, which are slow-relaxing impurity, rapidly relaxing impurity, and valence exchange [53], can be excluded in our case since all these processes are characterized by a strong temperature dependence, which we did not find in our experiments.

It is worth noting that even at high magnetic fields the observed damping of the spin oscillations still surpasses that reported for $(\mathrm{Ga}, \mathrm{Mn}) \mathrm{As}$. The reason for such a large damping can be due to band edge alignment of the present structure, imposing electronic potential fluctuations on top of the above mentioned magnetic inhomogeneities. Additionally note that the strength of the spin-orbit interaction is expected to be stronger for (In,Mn)As than that for $(\mathrm{Ga}, \mathrm{Mn}) \mathrm{As}$, which may contribute to the damping. It is noteworthy that decreasing of the oscillations dephasing time due to the $g$-factor distribution does not play a role since one should assume dispersion of the $\mathrm{Mn}^{2+}$ ions $g$ factor as large as 4 .

To conclude, we performed systematic studies of the laser-induced magnetization dynamics in ( $\mathrm{In}, \mathrm{Mn}) \mathrm{As}$ at temperatures down to $1.6 \mathrm{~K}$ and in magnetic fields up to $7 \mathrm{~T}$. Laser-induced ferromagnetic spin precession was observed and it was found that the effective damping of the magnetic oscillations in ( $\mathrm{In}, \mathrm{Mn}) \mathrm{As}$ is much larger than that in $(\mathrm{Ga}, \mathrm{Mn})$ As. A strong magnetic field dependence of the effective damping was discovered, which is attributed to the compositional randomness resulting in the presence of the paramagnetic regions even below the Curie temperature. The latter hampers ultrafast laser excitation of the ferromagnetic resonance in this ferromagnetic semiconductor and can explain the observed magnetic field dependence of the effective damping.

A Toonen, A. van Roij, and A. van Etteger are acknowledged for indispensable technical support during the experiments. R.R.S. acknowledges R. V. Mikhaylovskiy, J. Becker, and D. Afanasev for fruitful discussions and suggestions. R.R.S., A.V.K., and T.R. acknowledge the European Community's Seventh Framework Programme FP7NMP-2011-SMALL-281043 (FEMTOSPIN), the European Research Council ERC Grant Agreement No. 257280 (Femtomagnetism), the Foundation for Fundamental Research on Matter (FOM), as well as the Netherlands Organization for Scientific Research (NWO). H.M. acknowledges supports in part by the Advanced Photon Science Alliance Project from MEXT and Grand-in-Aid for Scientific Research No. 22226002 from JSPS, and N. Nishizawa for discussions concerning magnetic characterization. 
[1] K. S. Burch, D. D. Awschalom, and D. N. Basov, J. Magn. Magn. Mater. 320, 3207 (2008).

[2] T. Dietl, Nat. Mater. 9, 965 (2010).

[3] H. Munekata, in Concepts in Spin Electronics, edited by S. Maekawa (Oxford Science, Oxford, 2006), pp. 1-42.

[4] F. Matsukuraa, H. Ohno, and T. Dietla, in Handbook of Magnetic Materials, edited by K. H. J. Buschow (Elsevier, Amsterdam, 2002), pp. 1-87.

[5] P. Kacman, Semicond. Sci. Technol. 16, R25 (2001).

[6] T. Dietl, H. Ohno, and F. Matsukura, Phys. Rev. B 63, 195205 (2001).

[7] A. Oiwa, Y. Mitsumori, R. Moriya, T. Slupinski, and H. Munekata, Phys. Rev. Lett. 88, 137202 (2002).

[8] H. Ohno, D. Chiba, F. Matsukura, T. Omiya, E. Abe, T. Dietl, Y. Ohno, and K. Ohtani, Nature (London) 408, 944 (2000).

[9] D. Chiba, F. Matsukura, and H. Ohno, Appl. Phys. Lett. 89, 162505 (2006).

[10] H. Munekata and T. Schallenberg, Appl. Phys. Lett. 89, 042507 (2006).

[11] S. Ohya, I. Muneta, Y. F. Xin, K. Takata, and M. Tanaka, Phys. Rev. B 86, 094418 (2012).

[12] V. L. Korenev, I. A. Akimov, S. V. Zaitsev, V. F. Sapega, L. Langer, D. R. Yakovlev, Y. A. Danilov, and M. Bayer, Nat. Commun. 3, 959 (2012).

[13] I. Žutić, J. Fabian, and S. Das Sarma, Rev. Mod. Phys. 76, 323 (2004).

[14] S. Koshihara, A. Oiwa, M. Hirasawa, S. Katsumoto, Y. Iye, C. Urano, H. Takagi, and H. Munekata, Phys. Rev. Lett. 78, 4617 (1997)

[15] G. V. Astakhov, H. Hoffmann, V. L. Korenev, T. Kiessling, J. Schwittek, G. M. Schott, C. Gould, W. Ossau, K. Brunner, and L. W. Molenkamp, Phys. Rev. Lett. 102, 187401 (2009).

[16] G. V. Astakhov, J. Schwittek, G. M. Schott, C. Gould, W. Ossau, K. Brunner, and L. W. Molenkamp, Phys. Rev. Lett. 106, 037204 (2011).

[17] A. Oiwa, H. Takechi, and H. Munekata, J. Supercond. 18, 9 (2005).

[18] D. M. Wang, Y. H. Ren, X. Liu, J. K. Furdyna, M. Grimsditch, and R. Merlin, Phys. Rev. B 75, 233308 (2007).

[19] Y. Hashimoto, S. Kobayashi, and H. Munekata, Phys. Rev. Lett. 100, 067202 (2008).

[20] E. Rozkotova, P. Nemec, P. Horodyska, D. Sprinzl, F. Trojanek, P. Maly, V. Novak, K. Olejnik, M. Cukr, and T. Jungwirth, Appl. Phys. Lett. 92, 122507 (2008).

[21] J. Qi, Y. Xu, A. Steigerwald, X. Liu, J. K. Furdyna, I. E. Perakis, and N. H. Tolk, Phys. Rev. B 79, 085304 (2009).

[22] A. V. Scherbakov, A. S. Salasyuk, A. V. Akimov, X. Liu, M. Bombeck, C. Bruggemann, D. R. Yakovlev, V. F. Sapega, J. K. Furdyna, and M. Bayer, Phys. Rev. Lett. 105, 117204 (2010).

[23] P. Nemec, E. Rozkotova, N. Tesarova, F. Trojanek, E. De Ranieri, K. Olejnik, J. Zemen, V. Novak, M. Cukr, P. Maly, and T. Jungwirth, Nat. Phys. 8, 411 (2012).

[24] N. Tesarova, P. Nemec, E. Rozkotova, J. Zemen, T. Janda, D. Butkovicova, F. Trojanek, K. Olejnik, V. Novak, P. Maly, and T. Jungwirth, Nat. Photon. 7, 493 (2013).

[25] G. A. Khodaparast, D. C. Larrabee, J. Kono, D. S. King, J. Kato, T. Slupinski, A. Oiwa, H. Munekata, G. D. Sanders, and C. J. Stanton, J. Appl. Phys. 93, 8286 (2003).
[26] M. Bhowmick, T. R. Merritt, G. A. Khodaparast, B. W. Wessels, S. A. McGill, D. Saha, X. Pan, G. D. Sanders, and C. J. Stanton, Phys. Rev. B 85, 125313 (2012).

[27] J. G. Wang, C. J. Sun, Y. Hashimoto, J. Kono, G. A. Khodaparast, L. Cywinski, L. J. Sham, G. D. Sanders, C. J. Stanton, and H. Munekata, J. Phys.: Condens. Matter 18, R501 (2006).

[28] H. Munekata, Mat. Sci. Eng. B Solid 31, 151 (1995).

[29] H. Ohno, H. Munekata, T. Penney, S. von Molnar, and L. L. Chang, Phys. Rev. Lett. 68, 2664 (1992).

[30] T. Jungwirth, J. Sinova, J. Masek, J. Kucera, and A. H. MacDonald, Rev. Mod. Phys. 78, 809 (2006).

[31] M. Dobrowolska, K. Tivakornsasithorn, X. Liu, J. K. Furdyna, M. Berciu, K. M. Yu, and W. Walukiewicz, Nat. Mater. 11, 444 (2012).

[32] V. F. Sapega, M. Ramsteiner, O. Brandt, L. Daweritz, and K. H. Ploog, Phys. Rev. B 73, 235208 (2006).

[33] V. I. Litvinov and V. K. Dugaev, Phys. Rev. Lett. 86, 5593 (2001).

[34] A. V. Kimel, F. Bentivegna, V. N. Gridnev, V. V. Pavlov, R. V. Pisarev, and T. Rasing, Phys. Rev. B 63, 235201 (2001)

[35] S. A. Crooker, D. D. Awschalom, J. J. Baumberg, F. Flack, and N. Samarth, Phys. Rev. B 56, 7574 (1997).

[36] X. Y. Liu and J. K. Furdyna, J. Phys.: Condens. Matter 18, R245 (2006).

[37] X. Liu, W. L. Lim, Z. Ge, S. Shen, M. Dobrowolska, J. K. Furdyna, T. Wojtowicz, K. M. Yu, and W. Walukiewicz, Appl. Phys. Lett. 86, 112512 (2005).

[38] X. Liu, W. L. Lim, M. Dobrowolska, J. K. Furdyna, and T. Wojtowicz, Phys. Rev. B 71, 035307 (2005).

[39] Z. Q. Yuan, R. R. Du, M. J. Manfra, L. N. Pfeiffer, and K. W. West, Appl. Phys. Lett. 94, 052103 (2009).

[40] R. Winkler, D. Culcer, S. J. Papadakis, B. Habib, and M. Shayegan, Semicond. Sci. Technol. 23, 114017 (2008).

[41] J. Wang, C. Sun, J. Kono, A. Oiwa, H. Munekata, L. Cywinski, and L. J. Sham, Phys. Rev. Lett. 95, 167401 (2005).

[42] A. V. Kimel, A. Kirilyuk, P. A. Usachev, R. V. Pisarev, A. M. Balbashov, and T. Rasing, Nature (London) 435, 655 (2005).

[43] A. M. Kalashnikova, A. V. Kimel, R. V. Pisarev, V. N. Gridnev, A. Kirilyuk, and T. Rasing, Phys. Rev. Lett. 99, 167205 (2007).

[44] V. I. Belotelov and A. K. Zvezdin, Phys. Rev. B 86, 155133 (2012).

[45] M. Farle, Rep. Prog. Phys. 61, 755 (1998).

[46] J. Wang, L. Cywinski, C. Sun, J. Kono, H. Munekata, and L. J. Sham, Phys. Rev. B 77, 235308 (2008).

[47] L. Cywinski and L. J. Sham, Phys. Rev. B 76, 045205 (2007),

[48] M. Abolfath, T. Jungwirth, J. Brum, and A. H. MacDonald, Phys. Rev. B 63, 054418 (2001).

[49] K. Hamaya, T. Watanabe, T. Taniyama, A. Oiwa, Y. Kitamoto, and Y. Yamazaki, Phys. Rev. B 74, 045201 (2006).

[50] A. Oiwa, T. Slupinski, and H. Munekata, Appl. Phys. Lett. 78, 518 (2001).

[51] M. Berciu and R. N. Bhatt, Phys. Rev. Lett. 87, 107203 (2001).

[52] K. Hamaya, T. Taniyama, Y. Kitamoto, T. Fujii, and Y. Yamazaki, Phys. Rev. Lett. 94, 147203 (2005).

[53] M. Sparks, Ferromagnetic-Relaxation Theory, McGraw-Hill Advanced Physics Monograph Series (McGraw-Hill, New York, 1964). 